\title{
Wavepacket intermittency and its role in turbulent jet noise
}

\author{
Oliver T. Schmidt, Tim Colonius \\ California Institute of Technology, Pasadena, CA, USA \\ Guillaume A. Brès \\ CASCADE Technologies, Palo Alto, CA, USA
}

\begin{abstract}
The intermittent behavior of large-scale coherent structures in turbulent jets is studied. These structures are the primary source of jet noise, and their emitted sound is in turn characterized by rapid amplitude modulations of the pressure field. These high-energy bursts are well portrayed in the frequency-time domain by means of time-local analysis techniques. Scaleograms obtained from wavelet transforms of a single-point pressure signals, for example, enable the identification of such loud events at specific locations. Our interest, however, is in the time-local behavior of the coherent structures as a whole to gain a physical understanding of jet noise generation. For that purpose, a time series of large-eddy simulation snapshots is projected onto two sets of modal basis functions that describe the large-scale structures in the frequency-domain. The first modal basis consists of frequency-domain, or spectral, POD modes that are empirically deduced from the data. The second basis is comprised of resolvent response modes that are obtained from a linear frequency-response analysis of the mean flow. The proposed method allows us to visualize the intermittent behavior of the modal solutions in the frequency-time domain in terms of magnitude contours of the projection coefficient. The results can then be interpreted in an analogous way to wavelet scaleograms. The limitations, benefits and the potential of the method to yield a low-order representation of the flow in the time domain are discussed.
\end{abstract}

\section{Introduction}

The spatial modulation of the large-scale coherent structures in turbulent jets makes them efficient sources of superdirective acoustic radiation to low emission angles (with respect to the jet axis). These coherent structures, often referred to as wavepackets, were shown to resemble spatial linear instabilities of the mean flow profile over a range of frequencies. ${ }^{1}$ Despite the good agreement in the near-field, jet noise models based on such modal solutions underpredict the sound radiated to the far-field significantly. An important aspect that is missing from these models is the temporal intermittency of the acoustic far-field. Simplified wavepacket models were shown to improve considerably when temporal variations of the amplitude and the spatial extent of the waveform are incorporated. ${ }^{2}$ They more closely mimic the noise of real jets, which is emitted in temporally localized burst. This behavior can be studied by time-local analysis techniques such as the wavelet transform. ${ }^{3}$

This paper aims at exploring the possibility to understand the intermittent behavior of large-scale coherent structures by analyzing them in the frequency-time domain. For this study, we use the large eddy simulation database of a Mach number $M=0.9$ and jet diameter Reynolds number $R e \approx 10^{5}$ turbulent jet computed by Brès at al. ${ }^{4}$ The visualization of the instantaneous streamwise perturbation velocity shown in figure 1 gives an idea of the wide range of spatial and temporal scales involved in the problem. Only the axisymmetric part of the fluctuation field is considered in this paper for brevity.

The paper is organized as follows. First, we compute two sets of basis functions in $\S$ II. A frequencydomain or spectral proper orthogonal decomposition (SPOD) based on short-time Fourier transforms of overlapping data segments allows us to construct an empirical basis. A second basis is obtained from a linear resolvent analysis of the mean flow. ${ }^{5-7}$ Both methods provide an orthogonal set of modes at any given

*oschmidt@caltech.edu 

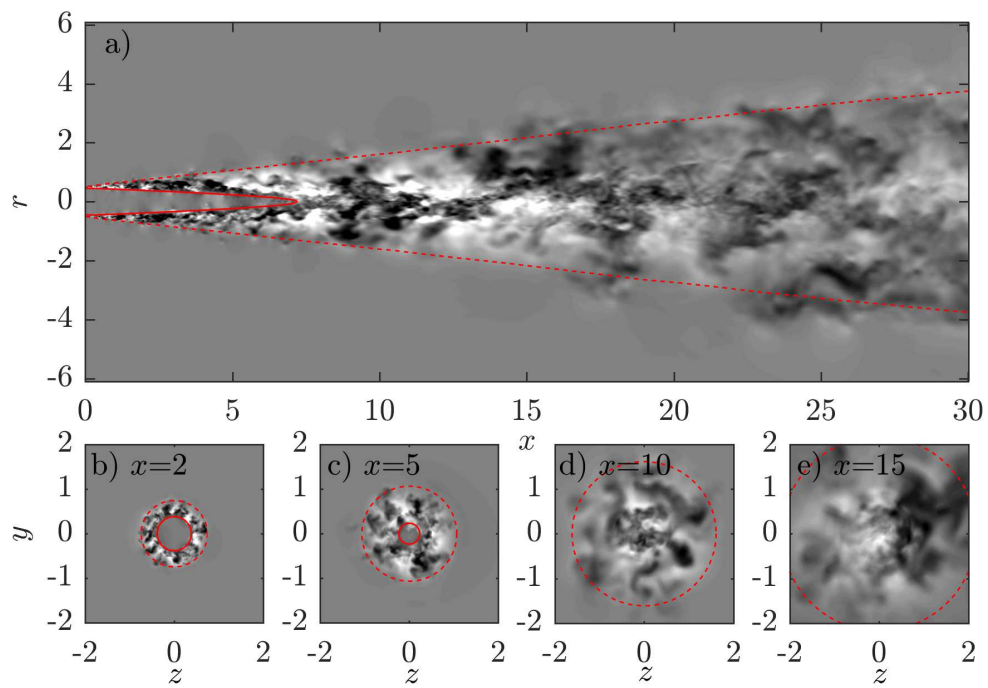

Figure 1. Instantaneous streamwise perturbation velocity ( $\left.\square \square,-0.5 \leq u_{x}^{\prime} /\left\|u_{x}^{\prime}\right\|_{\infty} \leq 0.5\right)$ and streamwise mean velocity $\left(-, \bar{u}_{x}=0.95 ;---, \bar{u}_{x}=0.05\right)$ of the LES: (a) streamwise plane and (b-e) transverse planes at $x=2,5,10$ and 15 , respectively.

frequency. In §III, we study the intermittency of the jet at different locations using a continuous wavelet transform of the pressure signal. An approach to examine the intermittency of the large-scale coherent structures in the frequency-time domain in introduced in $\S \mathrm{IV}$. It is based on projecting the simulation data onto the two sets of modes introduced in $\S I I$, and shares certain characteristics with the wavelet transform discussed in §III. Unlike the wavelet transform with is usually applied locally, our projection-based approach characterizes the time-local behavior of the entire flow field. Possible applications and extensions of the method are discussed in $\S \mathrm{V}$.

\section{Frequency-domain POD and resolvent response bases}

The jet is axisymmetric and the flow state $q=\left[\rho, u_{x}, u_{r}, u_{\theta}, T\right]^{T}(x, r, \theta, t)$ consisting of density $\rho$, cylindrical velocity components $u_{x}, u_{r}$ and $u_{\theta}$, and temperature $T$ can therefore be decomposed into azimuthal Fourier modes

$$
q(x, r, \theta, t)=\sum_{m} q_{m}(x, r, t) \mathrm{e}^{\mathrm{i} m \theta}
$$

of azimuthal wavenumber $m$. The unstructured LES data was interpolated onto a cylindrical grid with $N_{m}=128$ points in the azimuthal direction for that purpose. Under the assumption of statistical stationarity, we further decompose the data into spatio-temporal modes

$$
q_{m}(x, r, t)=\sum_{\omega} \tilde{q}_{m}(x, r) \mathrm{e}^{\mathrm{i} m \theta} \mathrm{e}^{\mathrm{i} \omega t}
$$

of angular frequency $\omega$. Snapshots were saved every $\Delta t=0.2$ acoustic units, resulting in a maximum Strouhal number of $S t=2.78$ for a Mach number of $M=0.9$. The most energetic coherent structures are educed from an ensemble of 10000 snapshots using frequency-domain or spectral proper orthogonal decomposition (SPOD)..$^{8,9}$ The SPOD is based on a short-time temporal Fourier transformation of $N_{b}=78$ blocks consisting of $N_{\mathrm{FFT}}=256$ snapshots with $50 \%$ overlap. Each block is regarded as an independent realization of the flow for estimation purposes. The ten most energetic modes were saved for each frequency. The column matrix of SPOD coefficients $\boldsymbol{\Phi}_{m}$ is obtained from an eigenvalue decomposition

$$
\boldsymbol{\Phi}_{m}^{H} \mathbf{Q}_{m}^{H} \mathbf{W} \mathbf{Q}_{m} \boldsymbol{\Phi}_{m}=\boldsymbol{\Lambda}_{m}
$$

of the column matrix of Fourier realizations $\mathbf{Q}_{m}=\left[\tilde{q}_{m}^{(1)}, \tilde{q}_{m}^{(2)}, \cdots, \tilde{q}_{m}^{\left(N_{b}\right)}\right]$. The basis of SPOD modes $\mathbf{\Psi}_{P O D}=$ $\mathbf{Q}_{m} \boldsymbol{\Phi}_{m} \boldsymbol{\Lambda}_{m}^{-1 / 2}$ optimally represent the data in terms of the total energy norm induced by the inner product

$$
\langle q, q\rangle_{E}=\iiint q^{H} \operatorname{diag}\left(\frac{\bar{T}}{\gamma \bar{\rho} M^{2}}, \bar{\rho}, \bar{\rho}, \bar{\rho}, \frac{\bar{\rho}}{\gamma(\gamma-1) \bar{T} M^{2}}\right) q r \mathrm{~d} x \mathrm{~d} r \mathrm{~d} \theta=q^{H} \mathbf{W} q
$$


where $\mathbf{W}$ is the discretized weight matrix associated with the norm, overbars denote mean quantities and $\gamma$ is the specific heat ratio. It was found that these most energetic coherent structures, of example identified via $\mathrm{POD}$ of microphone array measurement data, were similar to linear stability eigenfunctions obtained as PSE solutions. ${ }^{10}$

The second modal basis is obtained from a resolvent analysis ${ }^{7,11-13}$ based on the turbulent mean flow using the same numerical framework as described in Schmidt et al. ${ }^{14}$ The assumption of small perturbations of normal-mode form

$$
q^{\prime}(x, r, \theta, t)=q_{m}^{\prime}(x, r, t) \mathrm{e}^{\mathrm{i} m \theta}=\hat{q}_{m}(x, r) \mathrm{e}^{\mathrm{i}(m \theta-\omega t)}
$$

yields the spectral representation of the forced linearized governing equations

$$
\left(-\mathrm{i} \omega \mathbf{I}+\mathbf{L}_{m}\right) \hat{q}_{m}=\hat{f}_{m}, \quad \text { or } \quad \hat{q}_{m}=\left(-\mathrm{i} \omega \mathbf{I}+\mathbf{L}_{m}\right)^{-1} \hat{f}_{m}=\mathbf{R}_{m} \hat{f}_{m},
$$

where $\mathbf{L}_{m}=\mathbf{L}_{m}(\bar{q})$ is the discretized global linear stability operator representing the continuity, momentum, and energy equations for an ideal gas in cylindrical coordinates, and $\mathbf{R}_{m}=\mathbf{R}_{m}(\omega, \bar{q})=\left(-\mathrm{i} \omega \mathbf{I}+\mathbf{L}_{m}\right)^{-1}$ the global resolvent operator. If the time-harmonic forcing term $f^{\prime}=\hat{f}_{m} \mathrm{e}^{\mathrm{i}(m \theta-\omega t)}$ is identified with the non-linear interaction terms that were neglected during the linearization, equation (6) can be regarded as the spectral representation of the full governing equations. We are interested in forcing distributions that are optimal with respect to the energy gain

$$
\mu_{m}^{2}(\omega)=\max _{\hat{f}_{m}} \frac{\left\langle\hat{q}_{m}, \hat{q}_{m}\right\rangle_{E}}{\left\langle\hat{f}_{m}, \hat{f}_{m}\right\rangle_{E}}=\max _{\hat{f}_{m}} \frac{\left\langle\mathbf{R}_{m} \hat{f}_{m}, \mathbf{R}_{m} \hat{f}_{m}\right\rangle_{E}}{\left\langle\hat{f}_{m}, \hat{f}_{m}\right\rangle_{E}}=\max _{\hat{f}_{m}} \frac{\hat{f}_{m}^{H} \mathbf{R}_{m}^{H} \mathbf{W} \mathbf{R}_{m} \hat{f}_{m}}{\hat{f}_{m}^{H} \mathbf{W} \hat{f}_{m}}
$$

between forcings $\hat{f}_{m}$ and responses $\hat{q}_{m}$. The basis $\mathbf{F}_{m}=\left[\hat{f}_{m}^{(1)}, \hat{f}_{m}^{(2)}, \ldots, \hat{f}_{m}^{\left(N_{\lambda}\right)}\right]$ of optimal forcings is obtained from the eigenvalue decomposition $\mathbf{W}^{-1} \mathbf{R}_{m}^{H} \mathbf{W} \mathbf{R}_{m}=\mathbf{F}_{m} \boldsymbol{\Gamma}_{m}^{2} \mathbf{F}_{m}^{-1}$, where $\boldsymbol{\Gamma}_{m}^{2}=\operatorname{diag}\left(\mu_{m}^{2(1)}, \mu_{m}^{2(2)}, \ldots, \mu_{m}^{2\left(N_{\lambda}\right)}\right)$. The corresponding response basis is calculated as $\mathbf{Q}_{m}=\left[\hat{q}_{m}^{(1)}, \hat{q}_{m}^{(2)}, \ldots, \hat{q}_{m}^{\left(N_{\lambda}\right)}\right]=\mathbf{R}_{m} \mathbf{F}_{\mathbf{m}} \boldsymbol{\Gamma}^{-1}$, where $N_{\lambda}$ is the number of eigenvalues calculated by the Arnoldi algorithm. Both bases are orthogonal by construction and their constituting vectors are normalized to unit energy, i.e. $\mathbf{F}_{m}^{H} \mathbf{W} \mathbf{F}_{m}=\mathbf{Q}_{m}^{H} \mathbf{W} \mathbf{Q}_{m}=\mathbf{I}$.

A Reynolds number of $R e=3 \times 10^{4}$ is used for the frequency-response analysis to approximate the unknown effective Reynolds number of the turbulent flow. The computational domain of size $(x, r) \in$ $[0,30] \times[0,6]$ is discretized by $800 \times 200$ grid points. Grid points are clustered in the shear layer region and close to the nozzle in $r$ and $x$, respectively, and a sponge region is used to prevent numerical reflections from the boundaries. Ten resolvent forcing and response modes were saved at the same frequencies as for the SPOD, but limited to the interval $S t \in[0.1,1.5]$.
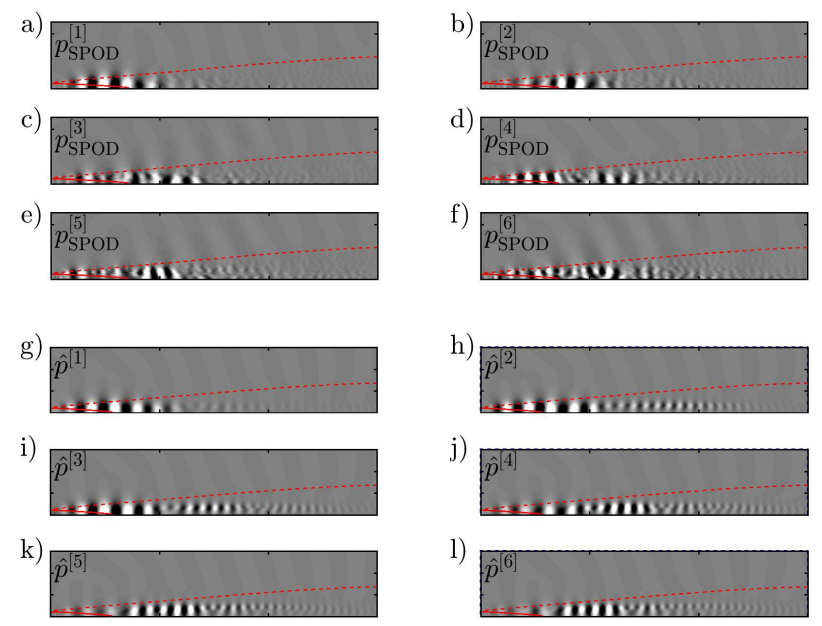

Figure 2. The six leading frequency-space POD modes (top three rows) and the six leading resolvent response modes (bottom three rows) for $S t=0.35$. The pressure is shown in $(x, r) \in[0,30] \times[0,6]$. The contour levels are capped at $50 \%$ of the maximum absolute value of each mode.

Figure 2 compares the six leading modes obtained from both methods. Both the leading SPOD modes and the leading resolvent response mode clearly resemble a Kelvin-Helmholtz-type instability of the initial 
shear-layer at this specific frequency. The higher order structures exhibit more complicated waveforms with modulated amplitude envelopes.
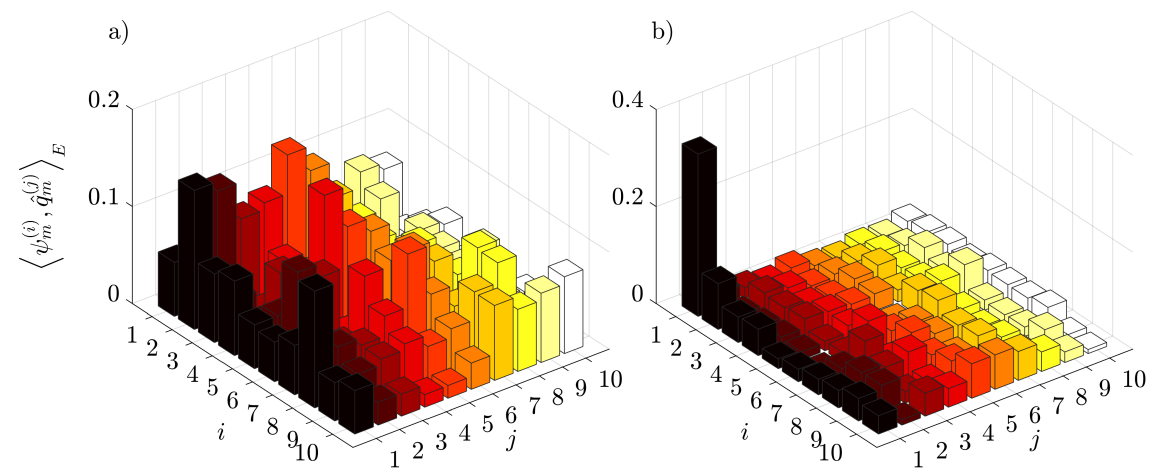

Figure 3. Projection of the frequency-space POD modes onto the resolvent response modes $\left\langle\psi_{m}^{(i)}, \hat{q}_{m}^{(j)}\right\rangle_{E}$ in the compressible energy norm: (left) $S t=0.2$; (right) $S t=0.6$.

The close resemblance between SPOD and resolvent response modes can be explained theoretically if certain properties hold for the forcing. ${ }^{9}$ Starting from the definition of the cross-special density matrices

$$
S_{\hat{q} \hat{q}}=\mathrm{E}\left\{\hat{q} \hat{q}^{H} \mathbf{W}\right\} \quad \text { and } \quad S_{\hat{f} \hat{f}}=\mathrm{E}\left\{\hat{f} \hat{f}^{H} \mathbf{W}\right\}
$$

for the state and the forcing, respectively, we can find a direct relation

$$
S_{\hat{q} \hat{q}}=\mathrm{E}\left\{\hat{q} \hat{q}^{H} \mathbf{W}\right\}=\mathrm{E}\left\{\mathbf{R}_{m} \hat{f} \hat{f}^{H} \mathbf{R}_{m}^{H} \mathbf{W}\right\}=\mathbf{R}_{m} \mathrm{E}\left\{\hat{f} \hat{f}^{H} \mathbf{W}\right\} \mathbf{R}_{m}^{H}=\mathbf{R}_{m} S_{\hat{f} \hat{f}} \mathbf{R}_{m}^{H}
$$

between the two quantities through the insertion of the definition of the resolvent (6). For forcing that is uncorrelated in space and time with $S_{\hat{f} \hat{f}}=\mathbf{I}$, the response CSD can be expressed solely in terms of the resolvent operator as

$$
S_{\hat{q} \hat{q}}=\mathbf{R}_{m} S_{\hat{f} \hat{f}} \mathbf{R}_{m}^{H}=\mathbf{R}_{m} \mathbf{R}_{m}^{H}=\mathbf{Q}_{m} \boldsymbol{\Gamma}_{m} \mathbf{F}_{m}^{H} \mathbf{F}_{m} \boldsymbol{\Gamma}_{m}^{H} \mathbf{Q}_{m}^{H}=\mathbf{Q}_{m} \boldsymbol{\Gamma}_{m}^{2} \mathbf{Q}_{m}^{H} .
$$

The last equality of this expression shows that the resolvent response modes coincide with the eigenvectors of $S_{\hat{q} \hat{q}}$, and therefore with the SPOD modes. This correspondence can be addressed qualitatively by projecting the modes onto each other. Figure 3 shows the mutual projection for a representative low and intermediate frequency. At $S t=0.6$, the first SPOD and leading resolvent modes agree favourably. This observation is likely related to the preferred amplification behavior of the jet. ${ }^{5}$ At lower frequencies such as $S t=0.2$, no such clear correspondence is observed. Consequently, a low-order representation of the most energetic SPOD mode in the span of only a few leading resolvent modes is not feasible.

\section{Wavelet transformation}

In the following, a frequency-time analysis based on a continuous wavelet transform is conducted. We use the analytic Morse wavelet with with zero demodulate skewness and a standard time-bandwidth product of 60 .

Figure 4 shows the temporal evolution of the wavelet coefficient of the pressure signal at $(x, r)=(19,6)$ for a pseudo-Strouhal number of $\tilde{S} t=0.2$. These parameters correspond to the location of the maximum overall sound pressure level in the far-field and the frequency of the peak power spectral density. The envelope modulation of the wavelet coefficient clearly indicates that the far-field noise is comprised of a series of loud events. The corresponding probability density function of the coefficient is well approximated by a Gaussian distribution.

The full scaleogram for the same location is shown in 5 (top, left). As discussed by Koenig et al., ${ }^{3}$ the highest acoustic activity is observed for $\tilde{S} t \approx 0.2$ in the form of high-energy bursts. The power spectral density (PSD) of the pressure signal (top, right) peaks at that frequency. The PSD is estimated from the pressure signal using Welch's method with 256 data points per segment and an overlap of $50 \%$ (in accordance with 

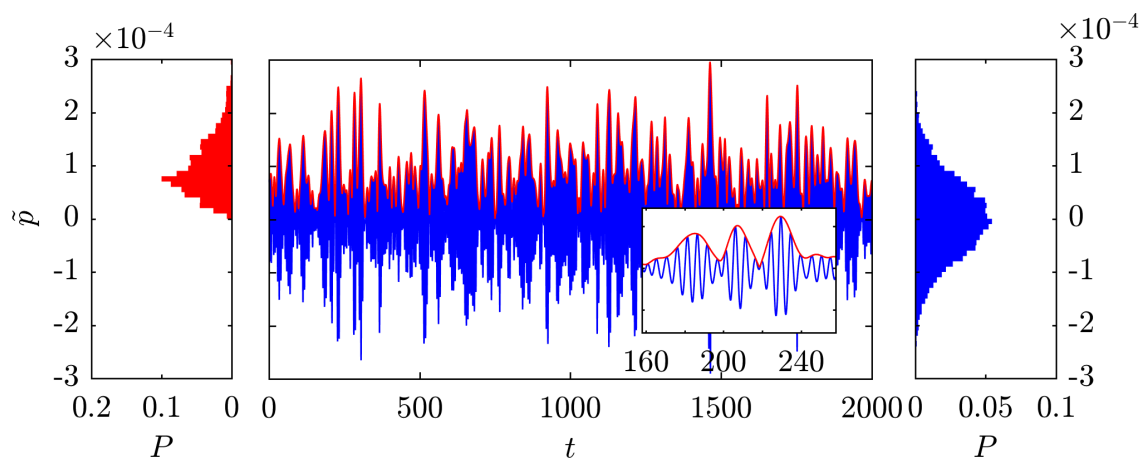

Figure 4. Wavelet transformation of the pressure signal at $(x, r)=(19,6)$ for $\tilde{S t}=0.2$ : (middle) time evolution of the wavelet coefficient (-) and its envelope (- - ; probability density function of the wavelet coefficient (right); probability density function of the wavelet coefficient envelope (left).
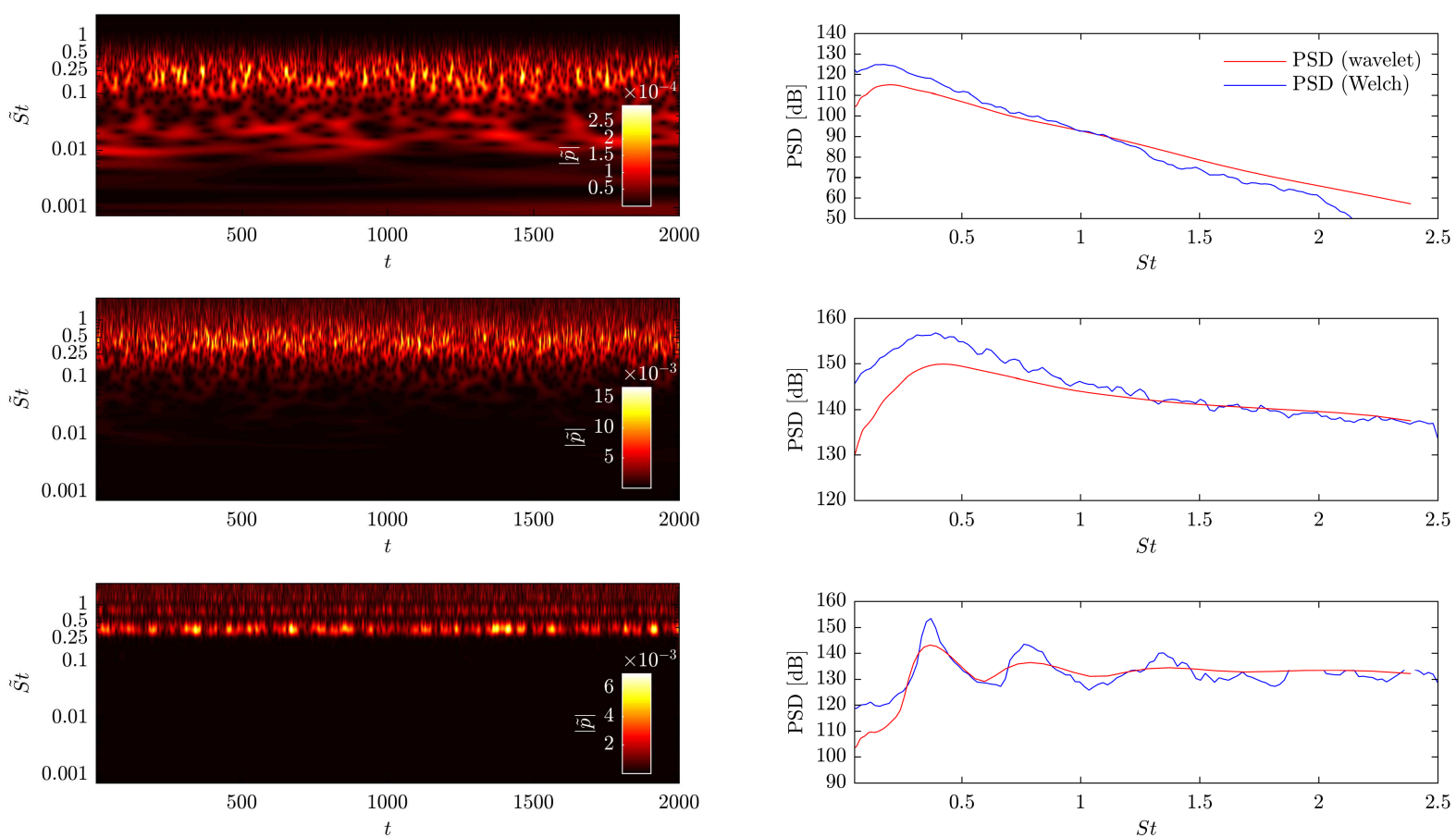

Figure 5. Scaleograms (left) and power spectral density (right) of the pressure at three representative locations: (top) far-field $(x, r)=(19,6)$; (middle) shear-layer $(x, r)=(4,0.5)$; (bottom) potential core $(x, r)=(0,0)$.

the SPOD, see $\S I I)$. Scaleograms and periodograms are also shown for two different locations $(x, r)=(4,0.5)$ (middle) and $(x, r)=(0,0)$ (bottom) that represent the shear-layer region and the potential core, respectively. The shear-layer region is characterized by a broad peak in the PSD around $\tilde{S} t \approx 0.4$ that is similarly reflected in the scaleogram. Three distinct energy bands are observed in the PSD and the scaleogram of the pressure in the nozzle plane on the jet axis. They are the energetic footprint of a class of trapped acoustic modes that are active in the potential core of high subsonic jets. ${ }^{14,15}$ Linear stability analyses describe these modes as neutrally stable or marginally stable. They have therefore be sustained by turbulent forcing. The resulting intermittent behavior is clearly reflected in the scaleogram. Approximations of the PSD from the wavelet coefficients (right, red lines) are also depicted in the periodograms and follow the major trends of the estimated PSD. 


\section{Data projection and modal expansion}

We start by establishing a notion of why it is useful to project time-domain data onto frequency-domain objects, i.e. to look at the inner products

$$
\left\langle\tilde{q}_{m}, \psi_{m}\right\rangle_{E}
$$

and

$$
\left\langle\tilde{q}_{m}, \hat{q}_{m}\right\rangle_{E}
$$

for the SPOD and the resolvent response bases, respectively. The SPOD modes calculated in $\S I I$ allow for an optimal low-order representation of flow data in the frequency-domain only, that is within the span of the $N_{b}=78$ Fourier mode realizations at any given frequency. Individual modes across different frequencies are not related by any orthogonality relation that could be exploited for an expansion of the time-dependent flow field. However, the underlying equations that govern the large-scale structures establish a fixed relation between frequency and spatial waveform. For less complex problems or locally, this property can often be expressed analytically in terms of a dispersion relation. For the complex problem at hand it is unknown, but can nevertheless be exploited, as discussed in the following. The conceptual idea is in that regard analogous to a wavelet transformation, where a mother wavelet is scaled in the time-domain in a way that it represents a pseudo-frequency in the spectral domain.

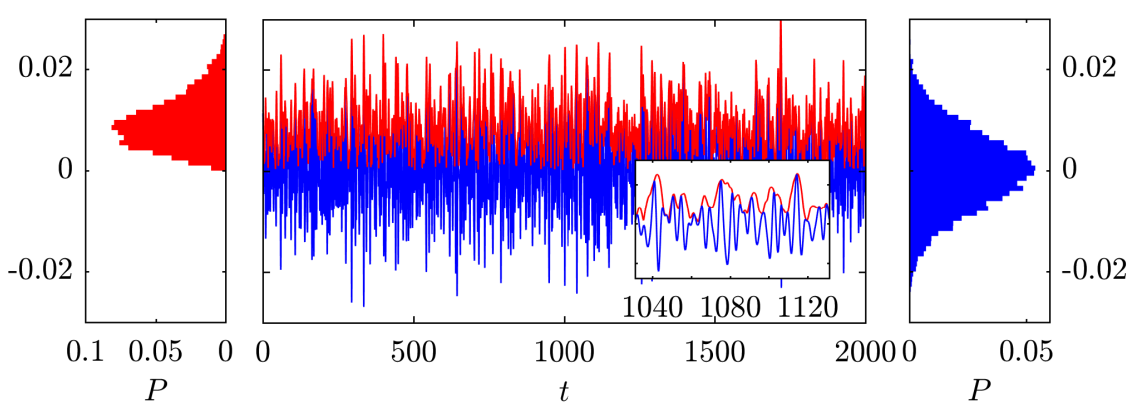

Figure 6. Real part of the projection $\left\langle\tilde{q}_{m}, \psi_{m}^{(1)}\right\rangle_{E}$ for the leading SPOD mode for $\tilde{\boldsymbol{S} t}=0.2$ and $m=0$ : (middle) time evolution of the projection coefficient $(-)$ and its envelope $(-)$; probability density function of the time signal (right); probability density function of the envelope (left).

To illustrate this property, the data $\tilde{q}_{m=0}(t)$ is projected onto the first SPOD mode $\psi_{m=0}^{(1)}$ for a specific frequency of $S t=0.2$. The real part of the resulting time series is plotted in figure 6 . It can be seen that the signal resembles a harmonic function that is modulated in time. The question whether the projection has the desired property, i.e. if the frequency of the harmonic oscillation corresponds to that of the SPOD mode, is addressed in the following.
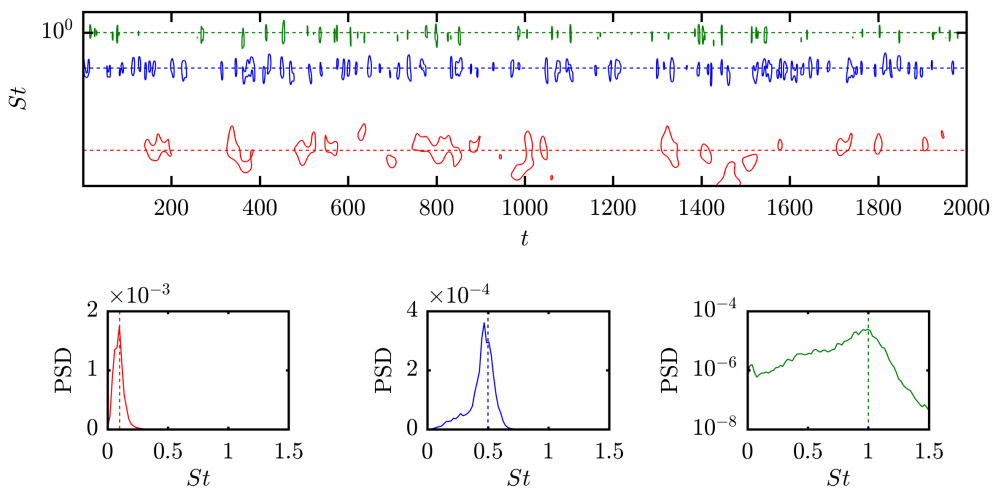

Figure 7. Scaleograms (top) and spectra (bottom) of the leading SPOD mode projection $\left\langle\tilde{q}_{m}, \psi_{m}^{(1)}\right\rangle_{E}$ for $S t=1(\longrightarrow)$, $S t=0.5(-), S t=0.1(-)$. The scaleogram contours correspond to $90 \%$ of the maximum absolute value of the wavelet coefficient.

In figure 7 , the data is projected onto the leading SPOD modes for three different frequencies. The resulting projection coefficient is analyzed in terms of a wavelet transform and PSD periodograms. It is 
observed that the time-local maxima of the wavelet coefficient magnitude are closely clustered around the frequency of the corresponding mode. Equivalently, the PSD peaks precisely at the expected frequency. This result demonstrates that the projection is an adequate measure for the temporal modulation of the individual structures. Similar results are obtained for the resolvent response modes.

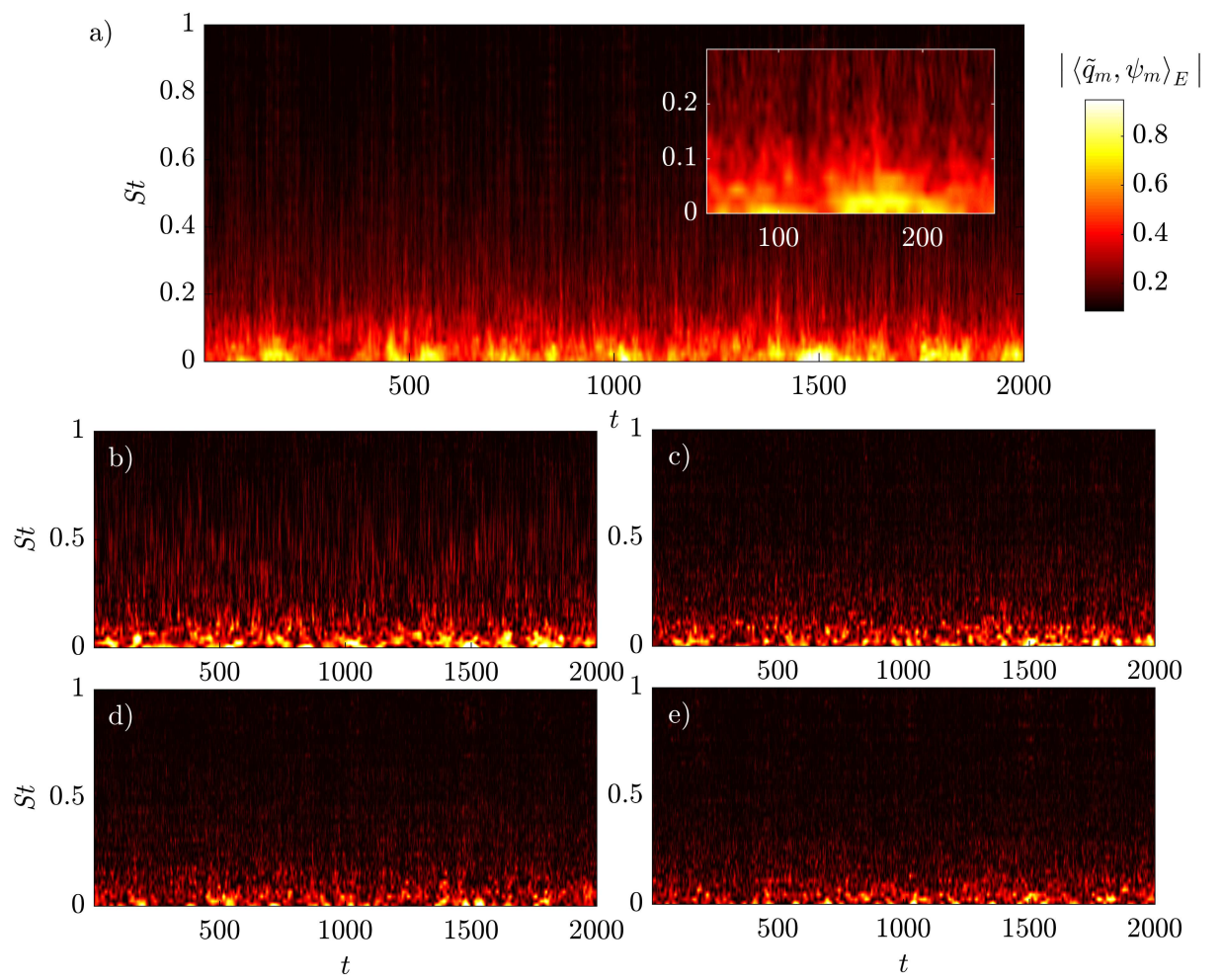

Figure 8. SPOD-based frequency-time diagram in terms of isocontours of $\left|\left\langle\tilde{q}_{m}, \psi_{m}\right\rangle_{E}\right|$ : (a) combination of 10 leading modes $\left|\Sigma_{i=1}^{10}\left\langle\tilde{q}_{m}, \psi_{m}^{(i)}\right\rangle_{E}\right|$; (b) optimal mode $\left|\left\langle\tilde{q}_{m}, \psi_{m}^{(1)}\right\rangle_{E}\right|$; (c-e) first three suboptimals $\left|\left\langle\tilde{q}_{m}, \psi_{m}^{(2,3,4)}\right\rangle_{E}\right|$, respectively.

We are now in the position to construct a frequency-time diagram similar to a scaleogram by projecting the data onto the entire ensemble of modes, and plotting the magnitude of the expansion coefficients over time. The frequency-time diagram can be constructed for a sum of modes for a more complete representation of the flow as in figure 8(a), or for individual modes as in figure 8(b-e). The optimality property of the SPOD modes facilities a low-order representation of the dynamics. Consequently, the projection coefficients of higher modes have a significantly lower magnitude, and the leading mode dominates the projection dynamics. Bursts of temporally localized activity are observed for very low frequencies. An example of such a high-energy event occurring at $150 \lesssim t \lesssim 210$ is highlighted in the insert of figure $8(\mathrm{a})$.

The resolvent modes are constructed such that they optimize the energetic gain between forcing and response modes. For that reason, the resolvent response modes do not necessarily represent the most energetic structures. It can therefore be expected that a larger number of modes is needed to capture the dynamics of the jet. The frequency-time diagram depicted in figure 9 (a) is constructed from the first 10 response modes and closely resembles the multiple-mode reconstruction based on SPOD seen in figure 8(a). The individual projections of the highest-gain mode and the three leading subdominant modes are shown in 9 (b) and 9 (ce), respectively. It can be seen that a change in the structure occurs at a frequency of $S t \approx 0.35$. Below this frequency the flow not properly approximated by just the leading resolvent mode. This observation is directly related to the relation between SPOD and resolvent modes, as discussed in the context of figure 3 . The difference becomes pronounced in the low frequency regime where the jet dynamics are not dominated by the preferred-amplification behavior, ${ }^{5}$ as previously discussed in the context of figure 3 .

This discussion would not be complete without addressing the question of whether the projection can be utilized for an expansion of the flow field in the time-domain. We address this question qualitatively by comparing the instantaneous pressure of the simulation to the modal reconstructions in figure 10 . It can be seen that the main features of the flow field 10(a) are captured by both the SPOD-based 10(b) and the resolvent-based reconstruction $10(\mathrm{c})$. Both the phase and the relative amplitude are matched accurately. 
a)
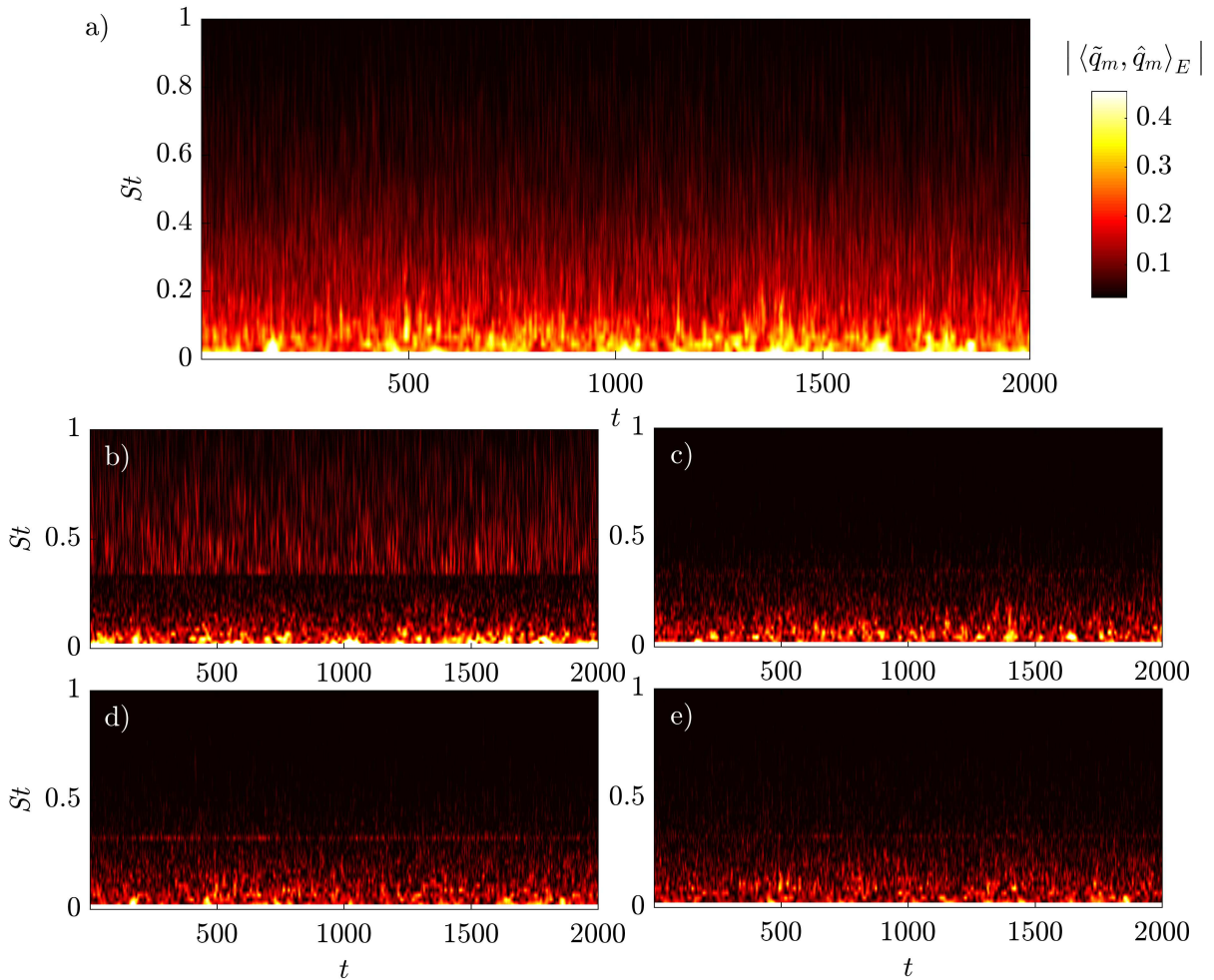

Figure 9. Resolvent-based frequency-time diagram in terms of isocontours of $\left|\left\langle\tilde{q}_{m}, \hat{q}_{m}\right\rangle_{E}\right|$ : (a) combination of 10 leading modes $\left|\Sigma_{i=1}^{10}\left\langle\tilde{q}_{m}, \hat{q}_{m}^{(i)}\right\rangle_{E}\right|$; (b) optimal mode $\left|\left\langle\tilde{q}_{m}, \hat{q}_{m}^{(1)}\right\rangle_{E}\right|$; (c-e) first three suboptimals $\left|\left\langle\tilde{q}_{m}, \hat{q}_{m}^{(2,3,4)}\right\rangle_{E}\right|$, respectively.

a)

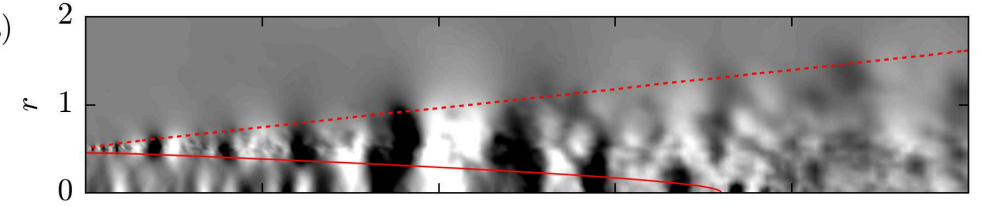

b)

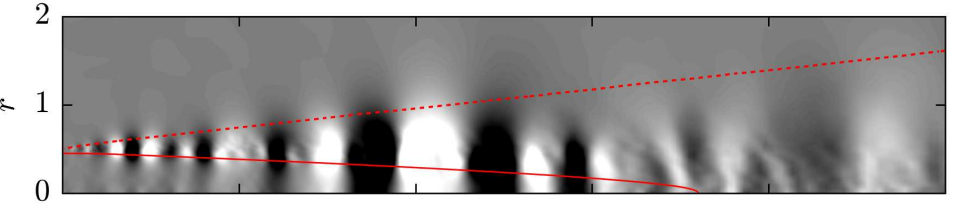

c)

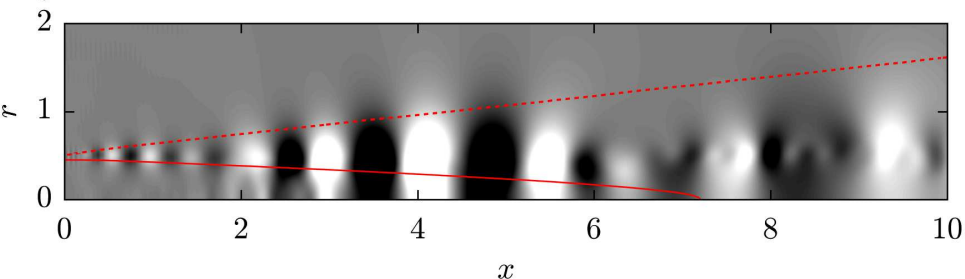

Figure 10. The instantaneous pressure field from the simulation (top) is compared to its reconstruction by the 6 leading frequency-space POD modes (middle) and the 10 leading resolvent response modes (bottom). The contour levels are capped at $25 \%$ of the maximum absolute value of each instantaneous field.

This result is notably for two reasons. First, the mathematical properties of the modes and their projection coefficients do not guarantee an accurate expansion. Second, both bases are restricted to a certain band of discrete frequencies and modes per frequency. An animation of the time history confirms that both expansions capture the intermittent dynamics of the large-scale coherent structures accurately within their respective span. 


\section{Discussion}

The suggested method enables a frequency-time domain analysis of large-scale coherent structures in turbulent flows. It is therefore well suited to study their intermittent behavior. An advantage over space-local wavelet transforms is that the resulting frequency-time diagrams contain information about the global dynamics, and that the suggested modal basis functions can be interpreted physically. An interesting prospect is to apply the method to flows that feature distinct non-linear effects such as vortex pairing or the generation of higher-harmonics by a dominant frequency. In the context of turbulent jets, the statistics of the projection coefficients can potentially be used to inform future low-order models that capture the intermittent characteristics of the flow field, and thus enable accurate predictions of jet noise. Future extensions of the method could include the use of optimization or regression techniques to find an optimal representation of the flow in the span of the chosen basis.

\section{Acknowledgments}

This research was supported in part by a grant from the Office of Naval Research (grant No. N0001416-1-2445) with Dr. Knox Millsaps as program manager. Oliver Schmidt was supported by DFG grant No. SCHM 3114/1-1. The LES study was performed at Cascade Technologies, with support from NAVAIR SBIR project, under the supervision of Dr. John T. Spyropoulos. The main LES calculations were carried out on DoD HPC systems in ERDC DSRC.

\section{References}

${ }^{1}$ Jordan, P. and Colonius, T., "Wave packets and turbulent jet noise," Annual Review of Fluid Mechanics, Vol. 45, 2013, pp. 173-195.

${ }^{2}$ Cavalieri, A. V. G., Jordan, P., Agarwal, A., and Gervais, Y., "Jittering wave-packet models for subsonic jet noise," Journal of Sound and Vibration, Vol. 330, No. 18, 2011, pp. 4474-4492.

${ }^{3}$ Koenig, M., Cavalieri, A., Jordan, P., Delville, J., Gervais, Y., Papamoschou, D., Samimy, M., and Lele, S., "Farfield filtering and source imaging for the study of jet noise," 16th AIAA/CEAS Aeroacoustics Conference and Exhibit, Vol. 3779, 2010, pp. 2010.

${ }^{4}$ Brès, G. A., Jaunet, V., Le Rallic, M., Jordan, P., Colonius, T., and Lele, S. K., "Large eddy simulation for jet noise: the importance of getting the boundary layer right," 21st AIAA/CEAS Aeroacoustics Conference, Vol. AIAA 2015-2535, Dallas, TX, 2015.

${ }^{5}$ Garnaud, X., Lesshafft, L., Schmid, P. J., and Huerre, P., "The preferred mode of incompressible jets: linear frequency response analysis," Journal of Fluid Mechanics, Vol. 716, 2013, pp. 189-202.

${ }^{6}$ Jeun, J., Nichols, J. W., and Jovanović, M. R., "Input-output analysis of high-speed axisymmetric isothermal jet noise," Physics of Fluids (1994-present), Vol. 28, No. 4, 2016, pp. 047101.

${ }^{7}$ McKeon, B. J. and Sharma, A. S., "A critical-layer framework for turbulent pipe flow," Journal of Fluid Mechanics, Vol. 658, Sep 2010, pp. 336382.

${ }^{8}$ Picard, C. and Delville, J., "Pressure velocity coupling in a subsonic round jet," International Journal of Heat and Fluid Flow, Vol. 21, No. 3, 2000, pp. 359-364.

${ }^{9}$ Towne, A., Colonius, T., Jordan, P., C. A., and Brès, G. A., "Stochastic and nonlinear forcing of wavepackets in a Mach 0.9 jet," 21st AIAA/CEAS Aeroacoustics Conference, 2015, p. 2217.

${ }^{10}$ Gudmundsson, K. and Colonius, T., "Instability wave models for the near-field fluctuations of turbulent jets," Journal of Fluid Mechanics, Vol. 689, 2011, pp. 97-128.

${ }^{11}$ Jovanović, M. R. and Bamieh, B., "Componentwise energy amplification in channel flows," Journal of Fluid Mechanics, Vol. 534, 2005, pp. 145-183.

${ }^{12}$ Sipp, D., Marquet, O., Meliga, P., and Barbagallo, A., "Dynamics and control of global instabilities in open-flows: a linearized approach," Applied Mechanics Reviews, Vol. 63, No. 3, 2010, pp. 030801.

${ }^{13}$ Monokrousos, A., Åkervik, E., Brandt, L., and Henningson, D. S., "Global three-dimensional optimal disturbances in the Blasius boundary-layer flow using time-steppers," Journal of Fluid Mechanics, Vol. 650, 2010, pp. 181-214.

${ }^{14}$ Schmidt, O. T., Towne, A., Colonius, T., Jordan, P., Jaunet, V., Cavalieri, A. V. G., and Brs, G. A., "Super- and multi-directive acoustic radiation by linear global modes of a turbulent jet," 22nd AIAA/CEAS Aeroacoustics Conference, American Institute of Aeronautics and Astronautics (AIAA), 2016.

${ }^{15}$ Towne, A., Cavalieri, A. V. G., Jordan, P., Colonius, T., Jaunet, V., Schmidt, O. T., and Brs, G. A., "Trapped acoustic waves in the potential core of subsonic jets," 22nd AIAA/CEAS Aeroacoustics Conference, American Institute of Aeronautics and Astronautics (AIAA), 2016. 